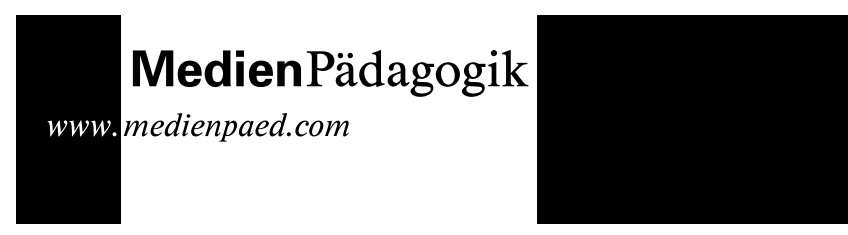

Andreas Breiter

6.11 .2002

\section{Wissensmanagementsysteme in Schulen oder: wie bringe ich Ordnung} ins Chaos?

Die zunehmende Ausstattung von Schulen mit digitalen Medien wird zu einer Herausforderung für die Organisationsentwicklung. Digitale Materialien der Lehrkräfte sowie Schülerarbeiten in digitalisierter Form wachsen tagtäglich an. Bisher fehlen geeignete organisatorische und technische Möglichkeiten zur Archivierung, Verteilung und Nutzung. In Unternehmen hat sich mit dem Begriff Wissensmanagement ein organsatorisches Konzept etabliert, das die Nutzung der Ressource Wissen optimieren will. Um diese Prozesse zu unterstützen, wurden und werden informations- und kommunikationstechnische Werkzeuge entwickelt, deren Übertragung auf den Schulbereich aufgrund dessen organisatorischer Komplexität aber scheitern muss. In diesem Beitrag werden diese Besonderheiten herausgestellt und die Erfahrungen mit der Entwicklung eines webbasierten Wissensmanagementsystems in Zusammenarbeit mit Schulen skizziert.

\section{Einleitung}

Die umfangreichen Ausstattungsinitiativen von Schulen mit neuen Medien durch Bund, Länder und Kommunen, die flankierenden Fortbildungsmassnahmen sowie die steigende Zahl von Softwareprodukten und OnlineAngeboten stellen neue Aufgaben an die organisatorische Integration. Die Zunahme an digitalen Unterrichtsmaterialien - sowohl aus eigenen Quellen (Vorbereitung der Lehrenden, Erarbeitung der Lernenden im Unterricht und zu Hause), als auch aus fremden Quellen (Lernsoftware und Nachschlagewerke auf CD-ROM, Internetquellen) - hat bereits in fortgeschrittenen Schulen einen Zustand erreicht, der eine Planung,
Organisation und Evaluation nach professionellen Methoden notwendig macht und ein Management der Wissenssammlung und des Wissensaustausch erfordert. Zugleich ist spätestens seit der Veröffentlichung der PISA-Ergebnisse deutlich geworden, dass dieser Austausch, d. h. die Kooperationsbeziehungen zwischen Lehrkräften unterentwickelt sind und eine breitere Verteilung und Transparenz des individuellen Fach- und Methodenwissens notwendig wird.

Seit Ende der 80er Jahre hat Wissen als Wettbewerbsfaktor in Unternehmen den Sprung in die Schlagzeilen geschafft. Hintergrund des gesteigerten Interesses ist die besondere Bedeutung des Wissens im Unternehmen. Während andere Ressourcen in Unternehmen nur noch begrenzt ausbaubar zu sein scheinen, gilt Wissen als die einzige Ressource, die sich durch den Gebrauch weiter vermehrt bzw. spezifiziert und damit die besondere Bedeutung eines Unternehmens bzw. die Nicht-Imitierbarkeit seiner Produkte begründen kann. Somit wird das Wissen zu einem strategischen Wettbewerbsfaktor. Der Begriff des Wissensmanagements lässt sich aus zweierlei Richtungen herleiten: einerseits gilt er als eine Erweiterung der Konzepte des Informationsmanagements bis hin zum Data Mining (informatikorientiert), andererseits als pragmatische Konkretisierung der Konzepte zur «lernenden Organisation» (organisationsbezogen).

Bildungseinrichtungen scheinen als «Häuser des Lernens» (Bildungskommission-NRW 1995) prädestiniert zu sein für Methoden zur Identifikation aller relevanten Informationsressourcen und Wissenspotenziale und ihrer systematischen Bereitstellung für alle Akteure. Welche der Verfahren unter welchen organisatorischen Bedingungen in Schulen wie technisch umgesetzt werden könnten, ist der Gegenstand dieses Beitrags. Damit wird eine besondere Bildungseinrichtung herausgegriffen für die erste Erfahrungen im Einsatz von technisch unterstützten Wissensmanagementsystemen vorliegen. Im Folgenden soll zuerst der theoretische Kontext dargestellt werden, aus dem sich die Konzepte des Wissensmanagements herausgebildet haben. Die organisatorischen Besonderheiten von Schulen und ihren Mitgliedern soll mit Hilfe des Modells der «Mülltonne»verdeutlicht werden. Am Beispiel eines von Informatikstudierenden ${ }^{1}$ gemeinsam mit Schulen entwickelten Prototyps werden die

${ }^{1}$ Der grösste Dank gilt allen 21 Studierenden im Projekt «LearnWeb» im Studiengang Informatik an der Universität Bremen (siehe www.learnweb-bremen.de) und den Lehrkräften aus der Integrierten Stadtteilschule am Leibnizplatz und an der Hermannsburg, dem Schulzentrum Bördestrasse und der Erwachsenenschule Bremen. 
Anforderung der Nutzerinnen und Nutzer an ein technisches System zusammengetragen und die Einsatzmöglichkeit unter Rückgriff auf den theoretischen Bezugsrahmen analysiert. Welche Erkenntnisse daraus für Schulen und andere Bildungseinrichtungen gezogen werden können, ist dann Gegenstand des Fazits.

\section{Informations- oder Wissensmanagement - oder doch besser:} lernende Organisationen?

2.1 Theoretische Konzepte des Informationsmanagements

In den 80er und 90er Jahren wurden in der Wirtschafts- und Verwaltungsinformatik Ansätze zu einem Informationsmanagement entwickelt, als organisatorische Lösungen für die gestiegene Bedeutung der Ressource Information. Damit war die Hoffnung verbunden, die gestiegene Komplexität aufgrund des raschen technologischen Wandels zu reduzieren und die dafür notwendigen Abstimmungsprozesse zu steuern. Ursprünglich stammt das Konzept aus den USA und richtete sich unter dem Begriff «Information Resource Management (IRM)» vor allem an Verwaltungsorganisationen, bei denen ein technischer Nachholbedarf gegenüber Unternehmen gesehen wurde (z. B. Synnott/Gruber 1981). Ziel des «[...] Informationsmanagements ist es, das Leistungspotential der Informationsfunktion für die Erreichung der strategischen Unternehmensziele durch die Schaffung und Aufrechterhaltung einer geeigneten Informationsinfrastruktur in Unternehmenserfolg umzusetzen» (Heinrich 1999, S. 21).

Bei den verschiedenen Ansätzen des Informationsmanagements lassen sich zwei grundsätzliche Sichtweisen erkennen. Auf der einen Seite wird versucht, ein normatives Konzept für eine Organisation zu definieren, wie diese mit der Ressource Information umgehen soll. Auf der anderen Seite geht es um einen analytischen Begriff, der die verschiedenen Aufgaben und Tätigkeiten umschreibt, die im Zusammenhang mit der Informationsverarbeitung in Unternehmen auftreten. In der Konsequenz findet sich in den meisten Lehrbüchern eine Vermischung der beiden Perspektiven - in der Regel wird das Informationsmanagement ausgehend von den normativen Kriterien begründet und die praktische Umsetzung sowohl aufbau- als auch prozessorientiert dargestellt.

Der Begriff Informationsmanagement lässt somit zahlreiche Interpretationen zu und scheint sich damit einer einheitlichen Definition zu entziehen (Krcmar 1991, S. 186f). Informationsmanagement ist ein diffuser Sammelbegriff unterschiedlicher Managementkonzeptionen, die sich auf unter- schiedliche Definitionen und Interpretationen der Begriffe «Information» und «Management» stützen. Mit Wollnik (1989) liesse sich zusammenfassend sagen, der Begriff Informationsmanagement sei eine modische Reaktion auf den Orientierungswandel in der Computerlandschaft, nicht ohne ernsthaften Bezug zur unternehmerischen Realität.

«In einen theoretischen Zusammenhang lässt er sich einbauen als Sammelbegriff für Reaktionstendenzen und Problemstellungen, die mit der Anwendungsreife, dem Leistungsvermögen und der wachsenden Anwendungsintensität moderner Informations- und Kommunikationstechniken entstanden sind. In praktischer Hinsicht kann er zum Ausgangspunkt einer Systematisierung von Einrichtungen und Vorkehrungen dienen, die sich auf Bereitstellung, Betrieb, aufgabenbezogene Anwendung und bestmögliche Nutzung der verfügbaren informations- und kommunikationstechnologischen Infrastrukturen richten.» (Wollnik 1989, S. 51)

Die theoretischen Ansätze werden zumeist in abstrakte Modelle (Aufgaben-, Ebenen- oder Architekturmodelle) gegossen und damit die mit dem Informationsmanagement verbundenen Aufgaben dargestellt. In diesen Modellen (für eine Übersicht siehe Krcmar 1996) geht es um verschiedene Managementaufgaben - Management von Informationen, Management von Informationssystemen und Management der Informations- und Kommunikationstechnik - die sich dann in strategische Ausrichtung, mittelfristige Planung und operative Umsetzung untergliedern lassen. Es geht um Aufgaben, die eher den klassischen Managementaufgaben entsprechen (wie Planung, Strategieentwicklung usw.), um Aufgaben, die sich mit der Umsetzung der Informationsflüsse im Unternehmen in ein technisches System einschliesslich der Infrastruktur beschäftigen, um Aufgaben, die eher techniknah mit der Anwendungsentwicklung und Nutzerunterstützung zu tun haben und um Aufgaben die unter Controlling subsumiert werden können.

\subsection{Theoretische Konzepte des organisationalen Lernens}

In der Literatur werden unter dem Begriff des organisationalem Lernens eigentlich zwei unterschiedliche Vorgänge bzw. Sachverhalte verstanden. Auf der einen Seite das individuelle Lernen, das Lernen und Weiterbilden von einzelnen Mitarbeiterinnen und Mitarbeitern für sich allein, und auf der anderen Seite das gemeinschaftliche, kollektive Lernen, das Lernen des Systems. Im Gegensatz zum individuellen Wissen ist organisationales Wissen nicht in den Köpfen von Menschen gespeichert, sondern in sozialen 
Systemen beziehungsweise in deren Regelsystemen (vgl. Geissler 1994). In den verschiedenen Konzepten des «Organisationalen Lernens» geht es um das grundlegende Verständnis der Rahmenbedingungen, Funktionsweisen und Hindernisse organisationaler Lernprozesse. Sie sind entstanden aus der Weiterentwicklung von Ansätzen der Organisationsentwicklung (OE), in denen bereits das (erfahrungsbezogenen) Lernen enthalten war (vgl. Argyris/Schön 1978). Allerdings bezogen sich OE-Konzepte auf den Lernprozess einzelner Organisationsmitglieder bzw. auf Gruppen und deren Qualifikation und Weiterbildung. Organisationales Lernen wird demgegenüber weiter gefasst. Neuere Theorien der Organisationsentwicklung haben nicht mehr das Gleichgewicht als Zielsetzung, sondern sie betonen die Entwicklungsdynamik als eigenes Lernpotenzial. Diese Dynamik erfordert innerhalb der organisatorischen Strukturen und Abläufe einen kontinuierlichen Lernprozess. Dabei geht es nicht um die lerntheoretisch fundierten Strategien der individuellen Verhaltensänderungen, sondern um Möglichkeiten, ganze soziale Systeme als aktiv lernende Gebilde zu betrachten (vgl. Pedler/Boydell/Burgoyne 1991, S. 61).

In der Fortführung seines Konzepts der kontinuierlichen Organisationsentwicklung beschreibt Senge das methodische Repertoire auf dem Entwicklungspfad eines Unternehmens zur lernenden Organisation. Insgesamt ermöglichen nach Senge fünf Disziplinen den Aufbau einer lernenden Organisation (Senge 1996). Sie umfassen vier Kerndisziplinen (Selbstführung und Persönlichkeitsentwicklung, mentale Modelle, gemeinsame Visionen und Teamlernen), die um das systemische Denken ergänzt werden. Systemisches Denken ist das Fundament der lernenden Organisation. Die individuelle Position insbesondere von Führungskräften darf nicht nur isoliert betrachtet werden, sondern es sind immer gleichzeitig Abhängigkeiten und Wechselwirkungen innerhalb des Systems zu bedenken und zu berücksichtigen.

Organisationales Lernen bedeutet daher zuallererst eine Modifikation der organisationalen Wissensbasis, z. B. über individuelles und teamorientiertes Lernen, über Speicherung und Kollektivierung von Wissen in der Organisation oder Transformation in Produkte und Dienstleistungen (vgl. Schüppel 1996). Hierbei stellen sich jedoch vielfältige Hürden: Lernprozessen immanent ist immer die Möglichkeit des Unerwarteten, der unvollständigen Kontrollierbarkeit und der tiefergreifenden Veränderung. Dadurch werden Organisationsstrukturen, Rollen und Funktionen in Frage gestellt, womit das Selbstbild einer Organisation und ihrer Akteure be- einflusst wird. Somit holen organisationale Lernprozesse Unsicherheit in eine Organisation und sind damit ein untypischer, eigentlich der Organisationslogik fremder Prozess. Es ist davon auszugehen, dass soziale Systeme dazu tendieren, gerade Unsicherheit zu vermeiden und Komplexität zu reduzieren und stattdessen Stabilität und Wiederholung präferieren (vgl. Baitsch 1993, S. 125). Diese Stabilität und Kontinuität kann nur mit der Etablierung organisationaler Routinen verwirklicht werden, da mit jeder aufgabenlogischen Wiederholung von Handlungsmustern die zukünftige Erwartungs- und Handlungssicherheit sichergestellt werden kann.

\subsection{Theoretische Konzepte des Wissensmanagements}

Aus den Ansätzen des organisationalen Lernens wird klar, dass der Weg zur lernenden Organisation bestimmter Instrumente bedarf, um ihn zu unterstützen. Vor dem Hintergrund des gesellschaftlichen und unternehmerischen Trends muss der Begriff «Wissensmanagement» für Organisationen gesehen werden als die Identifikation aller relevanten Wissenspotenziale und ihrer systematischen Ausschöpfung durch die Optimierung der Wissensflüsse entlang der Kernprozesse (vgl. Pulic 1996). Dass Organisationen überhaupt Wissen sammeln, speichern und verarbeiten können, wird immer wieder in Frage gestellt. Legt man die Definition von Wissen von Davenport und Prusak zugrunde, so wird die Beziehung zwischen individuellem und organisationalen Lernen deutlich: «Wissen ist eine fliessende Mischung aus strukturierten Erfahrungen, Wertvorstellungen, Kontextinformationen und Fachkenntnisse, die in ihrer Gesamtheit einen Strukturrahmen zur Beurteilung und Eingliederung neuer Erfahrungen und Informationen bietet. Entstehung und Anwendung von Wissen vollzieht sich in den Köpfen der Wissensträger. In Organisationen ist Wissen häufig nicht nur in Dokumenten oder Speichern enthalten, sondern erfährt auch eine allmähliche Einbettung in organisatorische Routinen, Prozess, Praktiken und Normen» (Davenport/Prusak 1998, S. 32).

Wissensmanagement lässt sich damit in den Kontext der pragmatischen Weiterentwicklung der Theorien und Perspektiven des organisationalen Lernens und des Informationsmanagements stellen. Im Zentrum des Interesses steht die Verbesserung der organisatorischen Fähigkeiten auf allen Ebenen der Organisation durch einen besseren Umgang mit der Ressource Wissen. Dafür wurden Instrumente entwickelt, die sich mit der Identifikation, dem Erwerb, der Entwicklung, Verteilung, Nutzung und Bewahrung von Wissen bemühen (z. B. Bullinger/Wörner/Prieto 1998). Im 
Kern des Wissensmanagement steht die Vorstellung, die unüberschaubare Menge an Daten und Informationen zu systematisieren und zu kategorisieren und das daraus entstandene Produkt unter Nutzung von Informationstechnologie in zeitlich und örtlich adäquater Form bereitzustellen. Dadurch soll es möglich werden, dass Nutzerinnen und Nutzer wettbewerbsrelevantes Wissen sowohl aus internen wie aus externen Quellen generieren und erwerben. Idealerweise könnte das an Personen gebundene Expertenwissen von den Trägern abgelöst und allgemein zur Verfügung gestellt werden.

Aus Sicht der Literatur zum Wissensmanagement besteht ein Unterschied zwischen implizitem Wissen («tacit knowledge»), das als Werkzeug für den Umgang mit dem im Blickpunkt stehenden Wissen dient und explizitem Wissen («focal knowledge»), Wissen über Objekte oder Phänomene, die gerade im Mittelpunkt stehen. Diese Unterscheidung geht zurück auf Polanyi (1966) und wurde von Nonaka und Takeuchi (1997) neu verwendet. Die Frage nach der Explizierung von Expertenwissen und ihre folgende Formalisierung, Modellierung und Digitalisierung hat die Forschung im Bereich der Künstlichen Intelligenz bereits Ende der 70er Jahre beschäftigt. Kritik gab es vor allem aus der philosophischen und soziologischen Sprachtheorie. Danach umfasst das Expertenwissen («Expertise») intuitives, nicht formalisierbares Wissen aus Lern- und Erfahrungsprozessen. Expertinnen und Experten folgen keinen Regeln, sie nutzen verstecktes Hintergrundwissen in speziellen situativen Kontexten (vgl. Suchman 1987; Dreyfus 1992). Ohne die sprachphilosophische Diskussion zu sehr zu strapazieren, bleibt festzuhalten, dass der Umgang mit, die Speicherung und Verarbeitung von Wissen bestimmten Restriktionen unterliegt, so dass von einem Wissensmanagement nur als einem Sammelbegriff für einen organisatorischen Veränderungsprozess gesprochen werden kann.

Der effiziente Umgang mit Wissen besteht oftmals darin, das Wissen anderer Menschen zu nutzen, ohne dieses Wissen zu erlernen. «Ein verbreiteter Kompromiss [...] besteht darin, dass beispielsweise nicht Wissensinhalte per se, sondern Wissensträger erfasst und der Organisation und deren Mitglieder allgemein zugänglich gemacht werden» (ReinmannRothmeier/Mandl 1999, S. 22). Um den Prozesscharakter zu betonen, sprechen Reinmann-Rothmeier vom Regelkreis des Wissensmanagements (Reinmann-Rothmeier/Mandl 1999, S. 19) bzw. Probst und andere noch umfassender von den acht Bausteinen des Managementkreislaufs (Probst/ Raub/Romhardt 1997, S. 58).

\begin{tabular}{|l|l|}
\hline Regelkreis des Wissensmanagements & Bausteine des Wissensmanagements \\
\hline - Wissensrepräsentation (Identifikation & $\bullet$ Wissensziele \\
von Wissen, Kodifizierung, & $\bullet$ Wissensidentifikation \\
Dokumentation und Speicherung) & $\bullet$ Wissenserwerb \\
- Wissenskommunikation (Verteilung, & $\bullet$ Wissensentwicklung \\
Vermittlung, Teilung und Ko- & $\bullet$ Wissensverteilung \\
Konstruktion und Kooperation) & $\bullet$ Wissensnutzung \\
- Wissensgenerierung (externe & $\bullet$ Wissensbewahrung \\
Beschaffung, Einrichtung von & $\bullet$ Wissensbewertung \\
Ressourcen, Schaffung personeller & \\
Netzwerke) & \\
- Wissensnutzung (Umsetzung in & \\
Entscheidungen und Handlungen sowie & \\
Transformation in Produkte und & \\
Dienstleistungen) & \\
\hline
\end{tabular}

Tabelle 1: Prozessbetrachtung des Wissensmanagements

In der Praxis ist eine Unterscheidung von Wissensmanagement gegenüber Informations-, Daten-, Hardware- oder Softwaremanagement nur schwer zu treffen (vgl. Schüppel 1996, S. 188). Das Wissensmanagement beschäftigt sich in erster Linie mit gestaltbaren - d. h. auch technisch modellierbaren Lernprozessen. In einer «organisationale Wissensbasis» (Datenbank) sind alle Elemente zusammengefasst, über die eine Organisation zur Lösung ihrer vielfältigen Aufgaben verfügt. Dazu zählen sowohl individuelle als auch kollektive Wissensbestandteile.

Am konsequentesten auf den Bildungsbereich ausgedehnt haben bisher Reinmann-Rothmeier und Mandl ihr Konzept. Für sie beinhaltet Wissensmanagement ein ganzes Bündel von Aufgaben in der Schule (vgl. Reinmann-Rothmeier/Mandl 1997, S. 20f):

- Informationen finden, aufnehmen, verarbeiten, reflektieren, bewerten;

- Informationen in Kontext einbetten, mit Bedeutung versehen, aus Informationen Wissen konstruieren;

- Wissensinhalte in einem kollektiven Gedächtnis abspeichern;

- Wissensinhalte an andere weitergeben, vermitteln, verteilen; 
- Wissensinhalte mit anderen kooperativ austauschen und gegenseitig ergänzen;

- Wissen anwenden und in Handeln umzusetzen;

- wissensbasiertes Handeln bewerten;

- das Erlernen der oben genannten Teilaufgaben des «Umganges» mit Information und Wissen;

- «Wissenspflege» (wie Aktualisierung, Eliminierung, Strukturierung) betreiben;

- neues Wissen «kreativ» entwickeln;

- Wissen transferieren und vieles mehr.

An diesen Aufgaben müssen sich alle informations- und kommunikationstechnischen Werkzeuge orientieren, wenn sie den Anspruch an eine adäquate Unterstützung der Anwenderinnen und Anwender haben.

\section{Schulen als soziale Organisationen}

Aus den theoretischen Ansätzen zum Wissensmanagement wird deutlich, dass es um mehr als die Modellierung von Daten und Informationen geht, sondern diese Prozesse in die Organisation eingebettet werden müssen und somit Wissensmanagement Teil der Organisationsentwicklung ist. In der Schulforschung wird sich schon seit Jahren mit der Organisationsentwicklung als Element der Schulentwicklung (neben Personal- und Unterrichtsentwicklung) beschäftigt (vgl. insbesondere Rolff u. a. 1998). Um die spezifischen Bedingungen von Schulen als komplexe soziale Organisationen zu verstehen und die damit verbundenen Rahmenbedingungen für ein Wissensmanagement zu verdeutlichen, ist ein Blick in die Organisationstheorie notwendig.

In Abgrenzung zu den rationalistischen Entscheidungstheorien der klassischen Organisationsforschung stammt von Cohen, March und anderen (Cohen/March/Olsen 1972) die Beschreibung der Entscheidungsarena in einer Organisation als Mülltonne («garbage can»), die insbesondere auf Universitäten bezogen war, sich aber auch auf Schulen übertragen lässt. Organisationen dieses Typs weisen folgende Merkmale auf (Cohen/ March/Olsen 1972, S. 2): Unklare Ziele, die erst durch Handlungen ermittelt werden, statt sie a priori zu setzen, unklare Verfahren, d. h. den Mitarbeiterinnen und Mitarbeitern sind die ablaufenden Prozesse und die Ursache-Wirkungs-Beziehung nicht deutlich, schwankende Beteiligung, sowohl zeitlich als auch inhaltlich.
Am Ende eines Entscheidungsprozesses stehen nicht selten Ergebnisse, die ursprünglich von niemandem intendiert waren, d. h. Entscheidungen werden eigentlich nicht getroffen, sondern oft erst im Nachhinein als solche rekonstruiert. Die Mülltonne enthält unsortiert verschiedene Elemente: Probleme bzw. Streitfälle, fertige Problemlösungen, Alternativen, Organisationsmitglieder mit wechselnden Zielen und plötzliche Situationsgelegenheiten für Entscheidungen. In der «organisatorischen Anarchie» herrscht in der Mülltonne eine hohe Dynamik, d. h. es können beliebige Elemente hinzukommen oder verschwinden bzw. sich wieder vermischen. Schaut man hinein, so gibt es Probleme, die eine Gelegenheit suchen nach oben zu kommen bzw. Streitfragen, die nach Klärung suchen. Die dank der arbeitsteiligen Struktur und durch zuvor festgelegte Koordinationsmechanismen bzw. -instanzen vorgefertigten Problemlösungen sind ebenfalls auf der Suche nach einem passenden Problem, das sie lösen können. In Organisationen existieren immer wieder Probleme, die nach einer Entscheidung suchen (sowohl routinemässig als auch neu und plötzlich), und es befinden sich zahlreiche Organisationsmitglieder in der Mülltonne mit unterschiedlichen wechselnden Zielen, die wiederum nach Problemen suchen oder an Entscheidungsfindungsprozessen teilnehmen wollen.

Für die Analyse des Innovationsprozesses in Schulen ist es zweckmässig, neben den vorherrschenden formalen Strukturen auch die informellen Entscheidungsprozesse auf der Akteursebene zu berücksichtigen. In Anlehnung an das Mülltonnen-Modell lassen sich die Beziehungen zwischen den Mitgliedern aus der Akteursperspektive auch als mikropolitische Entscheidungsprozesse betrachten, bei denen insbesondere Machtfragen eine Rolle spielen (Crozier/Friedberg 1979; Küpper/Ortmann 1988). Organisationsprozesse werden bei diesem Ansatz als Machtspiele interpretiert, «[...] deren formelle und informelle Regeln auf indirektem Wege den Zusammenhalt der widersprüchlichen mikropolitischen Strategien der Organisationsmitglieder bewirken» (Küpper/Ortmann 1988, S. 33). Macht wird dabei als gegenseitige Austauschbeziehung zwischen Akteuren verstanden, bei der sich die einen aufgrund von Vorteilen gegenüber anderen durchsetzen. Dabei spielen «[...] partielle Interessenskonvergenzen, zeitweise Koalitionen, Mauscheleien, Intrigen und Grabenkämpfe, side payments, bargaining-Prozesse, trojanische Pferde, Regimekritiker und Résistance, aber auch Übereifer, eigene Wünsche, Ängste und Konsensbedürfnisse [...]» (Küpper/Ortmann 1988, S. 35) eine gewichtige Rolle im Tauziehen um die Macht. Macht und Einfluss hängen somit 
sowohl von den formalen Regelungen, den Strukturen bzw. dem Zugang zu Ressourcen innerhalb einer Organisation ab, als auch von den Fähigkeiten zu ihrer Ausübung, von Know-how und Geschick. Die Machtspiele in einer Organisation wie der Schule lassen sich immer nur im Hinblick auf die spezifischen Bedingungen vor Ort und den Gegenstand der Entscheidungsprozesse genauer bestimmen.

Durch die Organisationsstruktur wird versucht, die verschiedenen Elemente in der Mülltonne und ihre Ströme zu kanalisieren (Aufgabenzuweisung, Ablaufsteuerung, Kompetenzverteilung usw.) - mit wechselhaftem Erfolg. Entscheidungen werden eher zu einem sozialen Ritual oder zu einem Abstimmungsprozess, der nicht immer mit den tatsächlichen oder intendierten Wirkungen zu tun hat, da es nur eine lose Kopplung zwischen den Elementen der Mülltonne gibt und die Lösungsverfahren und Tatbestände mehrdeutig interpretiert werden können (vgl. March/Olsen 1986).

Diese lose Kopplung als Schlüsselmerkmal wurde von Weick (1976) Organisationen zugeordnet, bei denen sowohl eine enge Verbindung zwischen den Elementen eines Systems als auch ein hoher Autonomiegrad existiert. Damit wird zum Ausdruck gebracht, dass solche Systeme sich durch eine hohe Selbständigkeit ihrer Einzelelemente auszeichnen, was zugleich eine geringe Steuerbarkeit und Berechenbarkeit wie auch eine hohe Flexibilität mit sich bringt. Steuerungsversuche in Bezug auf die Einzelelemente wirken sich nicht notwendigerweise auf das Gesamtsystem aus. Für Weick war das Schulsystem der idealtypische Fall eines lose-gekoppelten Systems.

Das Schulsystem lässt sich grundsätzlich als eine hierarchisch geordnete Bildungsverwaltung (äussere Organisation) mit einer ausgeprägten Binnendifferenzierung (innere Organisation) beschreiben, die sich in verschiedenen Schulformen, Schulzweigen, Bildungsgängen, Klassen usw. ausdrückt. Die Arbeitsteilung führt in der Schule zur Ausdifferenzierung in das pädagogische und das administrative Teilsystem. Innerhalb des pädagogischen Teilsystems gibt es weitere Untergliederungen in Fächergruppen, Schulstufen, Schulformen (sofern unterschiedliche Schulformen in einer Schule existieren) und Stellen für spezielle Aufgaben (Landkarten, Schulbibliothek, EDV usw.). Das administrative Teilsystem unterteilt sich weiter in Schulleitung, Fachbereichsleitung, Stufenleitung, deren Rolle sehr stark von der Struktur des Schulsystems und den etablierten Regeln und Verfahren in der Schule selbst abhängt. Auf der anderen Seite der starren Bürokratie steht eine relative Unabhängigkeit der Organisationsmitglieder untereinander, insbesondere hinsichtlich ihres beruflichen Auftrages, d. h. auf der Ebene der unmittelbaren Arbeit im Klassenzimmer verfügt die Lehrkraft über einen hohen Autonomiegrad. Die Bildungsbehörde hat die Personalhoheit und setzt die normativen Vorgaben (Rahmenpläne, Curricula, Schulgesetz, Standards), während für Lehrkräfte wenige Auflagen existieren und ein geringer Strukturierungs- und Formalisierungsgrad der schulischen Koordinationsformen und Kooperationsbeziehungen $\mathrm{zu}$ finden ist. Trotz der hierarchischen Struktur gibt es nur eine bedingte Kontrolle der Lehrkräfte, da Erfolg oder Misserfolg nur schwer zu bestimmen sind und die Aufsichtsspanne (ein Vorgesetzter auf etwa 100 Lehrkräfte) relativ gross im Vergleich zu Unternehmen ist. Von Fachgruppen abgesehen, gibt es in der Regel in Schulen keine Organisationsstrukturen, die zwischen den Kolleginnen und Kollegen sowie zwischen Kollegium und Schulleitung vermitteln. Kooperationen, wenn sie schulintern stattfinden, sind nur selten teamartig, sondern in der Regel gefügeartig (vgl. Rolff 1993). Sie sind an das Arbeitsgefüge der Schule gebunden, d. h. sie folgen in der vertikalen Arbeitsteilung der Trennung von Leitungs- und Lehrtätigkeiten; in der horizontalen Arbeitsteilung umfassen sie z. B. die Aufteilung der Lehrstoffe nach Fächern oder die Ordnung und Vermittlung der Inhalte nach Jahrgängen.

Nicht nur zwischen den Lehrkräften, sondern auch zwischen Schulen ein und derselben Kommune gibt es nur einen geringen Austausch - sie arbeiten auch nicht auf ein gemeinsames Ziel hin. Aber selbst zwischen den Schulstufen, die direkt aufeinander folgen (Grundschule und weiterführende Schulen, Sekundarstufe I und II) ist die Zusammenarbeit nur sehr begrenzt und reduziert sich häufig auf den Austausch bzw. das Ausleihen von Lehrkräften. Lortie spricht in diesem Zusammenhang von der «zellulären Grundstruktur der Schule», die sich im Klassenraum manifestiere (Lortie 1975, S. 13ff). Darunter versteht er die Inselbildung im Kollegium, die zu einer fehlenden Betrachtung der Schule als Einheit aus Sicht der Lehrkräfte führe, da bei ihnen die Klasse bzw. das Fach im Vordergrund stehe. Gerade diese funktionale Ausdifferenzierung in verschiedene Abteilungen (entweder fächerbezogen oder bei integrierten Schulen oder Schulzentren auch schulformbezogen) erschwert Kooperationen und macht Abstimmungsprozesse fast ausschliesslich in Form von Konferenzen bzw. von Anweisungen durch die Schulleitung oder durch die übergeordneten Behörden möglich. «In einem lose verknüpften Verbund teilautonomer Einheiten in einer Schule muss mit einer Vervielfachung von Subsystemen 
gerechnet werden, die wiederum eigene Grenzen, eigene Kulturen, eigene Rahmungen [...] an einer Schule ausbilden» (Warnken 1997, S. 23). Hinsichtlich des Einsatzes von Wissensmanagementsystemen oder kooperativen Lernsystemen sind aufgrund dieser organisatorischen Komplexität bereits strukturelle und prozessuale Rahmenbedingungen gesetzt, die es bei der Entwicklung und Implementierung zu berücksichtigen gilt.

\section{Umsetzung eines Wissensmanagementsystems für Schulen}

Der Hauptgrund, dass Wissensmanagement auf für Schulen ein Thema geworden ist, liegt in erster Linie an den Innovationszyklen bei der Informations- und Kommunikationstechnologie. Mit dem Trend zur technischen Vernetzung von Klassenzimmern entstehen Kommunikationsstrukturen, die mit traditionellen pädagogischen Organisationsmodellen nur schwer zu beschreiben sind. Tatsächlich scheinen es moderne Informationstechnologien wie Groupware-Applikationen oder Intranets zu sein, welche heute den Umgang der Schulen mit ihrer eigenen Wissensbasis verändern. Die Verbindung dieser technologischen Möglichkeiten mit dem Mensch und seinen individuell-einmaligen Fähigkeiten und Erfahrungen scheint der Haupttreiber in der Implementierung von Wissensmanagement zu sein.

Die Ziele eines Wissensmanagementsystems in Schulen liegen zuallererst im Organisationszweck begründet, d. h. der Verbesserung der Unterrichtsqualität durch den Einsatz digitaler Medien. Im Kern verschiedener Forschungsprojekte und Modellversuche steht die Frage, wie Schulen mit Wissen umgehen. Darunter lassen sich Aspekte der Lernkultur, des Wissensmanagements und der Kooperation und Kommunikation subsumieren. Durch die Integration neuer Medien in die Schule wird Schulentwicklung vorangetrieben. Damit ist als Folge bzw. auch als Voraussetzung zu erwarten, dass sich die Unterrichtsformen (projektorientiert, selbstgesteuert) Arbeitsformen (insbesondere Teamarbeit) und Organisationsformen (Stundenplangestaltung, Klassenraumausstattung) verändern bzw. weiter entwickeln müssen.

Aus Sicht von Chott besteht eine dreifache Notwendigkeit, sich mit Wissensmanagement zu beschäftigen (vgl. Chott 1998, S. 174ff): (1) bessere Vorbereitung der Schülerinnen und Schüler auf den Umgang mit Wissen (Methodenkompetenz); (2) Qualifizierung zum Aufnahme, zur Reflexion und zum kooperativer Austausch von Informationen und Wissen auf Gruppenebene der Lehrkräfte und (3) Konzepte zum Austausch von Wissen bei Schulentwicklungsprozessen.
4.1 Technische Realisierung im Rahmen eines studentischen Projektes mit Schulen

«Learnweb» ist der Name eines studentischen Hauptstudiumsprojektes im Studiengang Informatik der Universität Bremen. Ein wesentlicher Teil des Hauptstudiums (fünftes bis achtes Semester) ist die Teilnahme an einem Projekt. Diese Studienform geht auf die Gründungszeit der Bremer Universität zurück. Im Rahmen eines solchen Projektes sollen Studierende eine Aufgabenstellung analysieren, erlernte wissenschaftliche Methoden lösungsspezifisch anwenden und ihre Ergebnisse wissenschaftlichen Ansprüchen entsprechend darstellen. Die Themen werden von den Lehrenden vorgeschlagen, die Studierenden haben aber auf Thema, genaue Arbeitsvorhaben, auf die Art des Vorgehens und der Organisation Einfluss - je nachdem, wie sich die Verhältnisse in der konkreten Gruppe der beteiligten Menschen entwickeln. Typischerweise umfasst ein Projekt sechs bis acht Semesterwochenstunden.

Die Projektarbeit bietet über das Studium hinaus Gelegenheit, zahlreiche andere Fähigkeiten zu erwerben (wie Leitung von Sitzungen, Ergreifen von Initiativen, Durchsetzungsvermögen, Gruppen- und Teamarbeit, Kritik und Selbstkritik, Projektmanagement). Sie erfolgt in einem Team aus Lehrenden der Informatik und einer Anzahl von ungefähr 20 Studierenden. Das Projektstudium ist eine Studienform, die an wenigen Orten eine solch vielfältige und lang andauernde Tradition besitzt wie in Bremen. Oft kommen Studierende wegen dieser Form nach Bremen. Auch auf Seiten der Wirtschaft findet das Projektstudium aufgrund des hohen Praxisbezugs hohe Anerkennung.

Ziel des Projektes «LearnWeb» ist die Entwicklung, Erprobung und Evaluation einer webbasierten Plattform für Schulen. Dabei soll «LearnWeb» zwar eine Arbeitsumgebung zur Verfügung stellen, es grenzt sich aber bewusst von komplexeren Anwendungen wie «Computer Supported Cooperative Learning (CSCL)» ab (für eine Übersicht siehe Koschmann 1996). Die Aufgabe, die den Studierenden gestellt und die im Zuge der Anforderungsanalyse mit vier Modellschulen in Bremen konkretisiert wurde, zielt in die Richtung einer Organisations-, Interaktions- und Kommunikationsunterstützung für die schulischen Lehr- und Lernprozesse in Form eines Werkzeugs für das Informations- und Wissensmanagement innerhalb und ausserhalb der Schulen. Zielgruppe sind in erster Linie Lehrkräfte sowie Schülerinnen und Schüler, indirekt aber auch Eltern bzw. das Schulumfeld. Eine Verwandtschaft mit Werkzeugen des «Computer 
MedienPädagogik

Supported Cooperative Work (CSCW)» sind dabei sehr wohl beabsichtigt, das Entwicklungsziel bezog sich auf die schulspezifische Aufbau- und Ablauforganisation.

In jedem studentischen Projekt ist es Voraussetzung, den idealtypischen Softwareentwicklungsprozess mindestens einmal $\mathrm{zu}$ durchlaufen. Am Anfang des Projektes wurde darüber abgestimmt, welche Vorgehensweise präferiert werden sollte, um nicht zu enden wie in Abbildung 1 dargestellt.

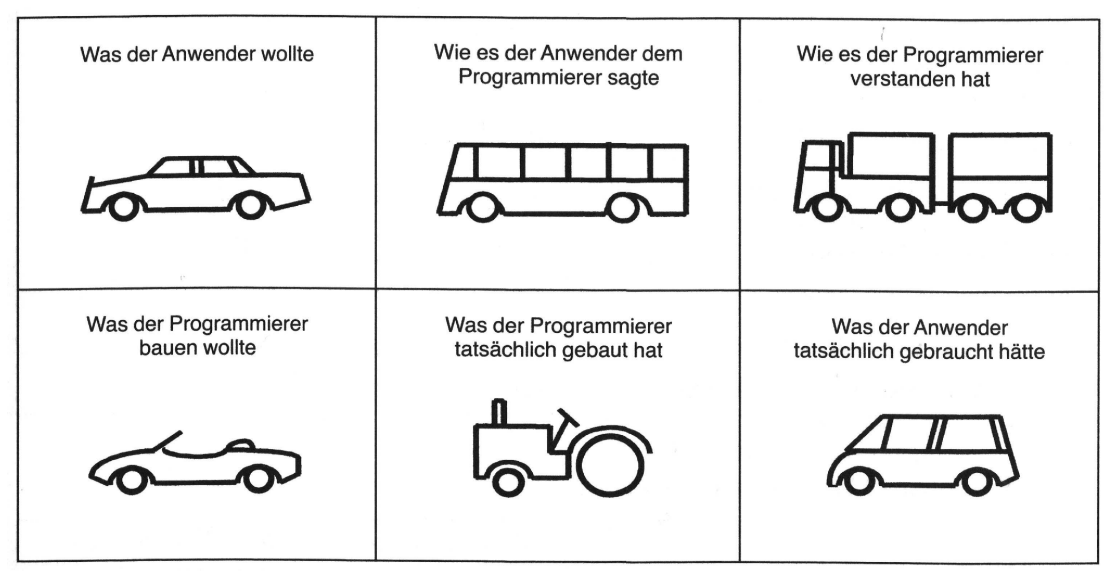

Abbildung 1: Anspruch und Wirklichkeit bei der Systementwicklung (Beer 2000, S. 21)

Aufgrund der Zielgruppe Schule, d. h. Lehrkräfte und Schülerinnen bzw. Schüler, wurde sich darauf verständigt, ein zyklisches Modell mit Prototypen zu verfolgen, um in regelmässigen Abständen die Zielgruppe nicht nur in den Entwicklungsprozess kommunikativ einzubeziehen, sondern anhand von konkreten Ergebnissen und Vorprodukten das weitere Vorgehen abzustimmen. In den verschiedenen Phasen des Projektes kamen sowohl horizontale als auch vertikale Prototypen zum Einsatz (für eine Unterscheidung siehe z. B. Balzert 1997, S. 114ff).

Bei der Konzeption eines Wissensmanagementsystems für Schulen müssen alle relevanten Akteure und ihre Kommunikations- und Kooperationsprozesse differenziert betrachtet werden:

- Schülerinnen und Schüler untereinander (klassenweise, AG-bezogen, schulweit, schulübergreifend),

- Lehrerinnen und Lehrer untereinander (klassenweise, fächerbezogen, AG-bezogen, schulweit, schulübergreifend),
- Lehrende und Lehrende miteinander (klassenweise, fächerbezogen, AG-bezogen, schulweit),

- Schulmitglieder (Lehrende und Lernende) mit Schulexternen (Eltern, lokales Umfeld, Schulbehörde)

\subsection{Anforderungsanalyse}

Ausgangspunkt für die Systementwicklung war eine intensive Beschäftigung mit Produkten, die bereits auf dem Markt erhältlich waren. Dabei wurden verschiedene Systeme getestet und auf den Bedarf von Schulen, auf die Portabilität bzw. Weiterentwicklungsmöglichkeiten und die Kosten hin bewertet. Bei den Bewertungskriterien wurde sich an verschiedenen bereits existierenden Studien orientiert (siehe v. a. Schulmeister 2000):

- Funktionale Anforderungen (Muss- und Kann-Kriterien),

- technische Anforderungen (Plattform, Bandbreiten, Rechtevergabe, Nutzerverwaltung, Webspace, Customizing, Weiterentwicklung),

- Use cases mit verschiedenen Szenarien aus Sicht von Lehrenden und Lernenden, Software-Ergonomie / Usability (GUI Design), Informationsinfrastruktur und

- finanzielle Aspekte.

Die frei verfügbaren Systeme wurden zusammen mit Schulen getestet, und es wurde auch mit den Entwicklern bei OpenSource-Produkten kommuniziert. Eine parallele Nutzung verschiedener Plattform ermöglicht eine wertvolle Erfahrungssammlung der Nutzerinnen und Nutzer bevor Systementscheidungen getroffen werden. Medien sind Erfahrungsgüter, deren Nutzen sich erst beurteilen lässt, wenn sie im Einsatz sind.

Auf Basis der Marktanalyse und den groben Anforderungen der Schulen wurde darauf verzichtet, existierende Produkte $\mathrm{zu}$ übernehmen und gegebenenfalls anzupassen, sondern es wurde eine Eigenentwicklung in enger Abstimmung mit den Schulen bevorzugt. Die Analyse brachte insbesondere fünf entscheidende Hürden zutage: Kosten, Administration, Weiterentwicklung, Kundenanpassung und Plattformunabhängigkeit. Da die Entwicklung für Schulen gedacht war und die finanzielle Situation der Schulträger eine teure Lösung nicht zulässt - zumal zum heutigen Zeitpunkt nicht einmal klar ist, dass sie auch genutzt würde, musste auf alle kommerziellen Lösungen verzichtet werden. Die Administration musste möglichst einfach für Lehrkräfte in der ihnen zur Verfügung stehenden Zeit zu erledigen sein, einige der Produkte, die bereits in Schulen eingesetzt werden, 
konnten diesem Anspruch nicht genügen. Die Weiterentwicklung bzw. Kundenanpassung lässt sich nur in den seltensten Fällen ohne zusätzliche Kosten gewährleisten. So steht dies prinzipiell bei OpenSource-Produkten offen, die Vorkenntnisse und die Einarbeitungszeit sind aber so hoch, dass dies nicht zu rechtfertigen ist. Die Frage nach der Plattformunabhängigkeit ist auch eine Spezialität Bremens, da hier noch eine grosse Anzahl Apple Macintosh in der Schulen vorhanden sind (ein Fünftel aller Geräte). Damit scheiden wiederum einige Produkte aus.

Die nachfolgende Anforderungsanalyse ist die am wenigsten technische Phase der Systementwicklung, aber nicht nur im Hinblick auf Schulen als Kunden, die wichtigste. Wenn sie nicht vollständig und adäquat durchgeführt wird, sind die Folgen gravierender als in den anderen Phasen, da die dort nicht gewonnenen Informationen später kaum rückholbar sind ohne den gesamten Prozess wieder von vorne zu starten. Auf der anderen Seite kann eine zu starke Berücksichtigung der variablen und sich dynamisch verändernden Interessen (Geschmäcker) der Endnutzerinnen und Endnutzer in der Konzeptionsphase zu Problemen und zu einer Verlangsamung des Entwicklungsprozesses führen, zumal sich einige Anforderungen kaum erfüllen lassen. Dennoch ist es mittlerweile Allgemeingut nicht nur in der partizipativen Systementwicklung (nach dem skandinavischen Modell, vgl. Bødker/Grønbæk 1991), dass die Folgen der Nichtberücksichtigung, Vernachlässigung, Missinterpretation oder gar Ignoranz der Nutzeranforderungen später kaum noch eingefangen werden können. Die Anforderungsanalyse erfordert soziale und kommunikative Fähigkeiten, die in der Regel bei Systementwicklern (hier Studierende der Informatik) nicht sehr stark ausgeprägt sind. Auf der anderen Seite sind Studierende der Sozialwissenschaften mit Kenntnissen von qualitativen Methoden überfordert für die Erfassung funktionaler Anforderungen an ein Softwaresystem. Daher werden in Bremen angehende Informatikerinnen und Informatiker darauf vorbereitet.

Innerhalb einer Anforderungsanalyse ist es notwendig, die erwarteten Leistungen und Einschränkungen zu definieren, die ein System bereitstellen bzw. denen es gehorchen muss. Alle Leistungen werden klassifiziert, um den vollen Umfang des Systems und dessen funktionale Eigenschaften und die dazugehörigen Datenstrukturen $\mathrm{zu}$ beschreiben. Die traditionelle Vorgehensweise bei der Systementwicklung endet mit der Anforderungsdefinition, in dem alle Anforderungen, die von den Nutzerinnen und Nutzern akzeptiert wurden, schriftlich niedergelegt sind (mit
Klassifikation, Nummer und Priorität). Um die Arbeitsprozesse von Lernenden und Lehrenden in den Schulen $\mathrm{zu}$ modellieren, wurden Unterrichtsbesuche, Gruppen- und Einzelinterviews durchgeführt. Die Resultate wurden mit Hilfe der Szenario-Methode (vgl. Carroll 1995) ermittelt, verschriftlicht und mit einer Modellierungssprache (UML - Unified Modelling Language) dargestellt.

Das grösste Problem der Anforderungsanalyse sind die Methoden der Identifikation und Explizierung der Anforderungen. Dabei besteht ähnlich wie beim Wissensmanagement - das Problem des versteckten Wissens auf der einen Seite und die Schwierigkeiten bei Systementwicklern mit qualitative Forschungsmethoden. Die Studierenden kombinierten hierfür - nach längeren Übungseinheiten - strukturierte und unstrukturierte Interviewtechniken mit offen und geschlossenen Fragen.

\subsection{Systementwicklung}

Die LearnWeb-Plattform ist eine reine Java-Applikation mit einer austauschbaren Benutzungsoberfläche. Für den Einsatz in Schulen war es notwendig, eine Oberfläche auf der Basis von HTML mit Java zu entwickeln, um das System webbasiert und damit plattformunabhängig zu gestalten. Die Benutzerinnen und Benutzer kommunizieren mit der Plattform über einen Internetbrowser mit Hilfe des http-Protokolls. Die Kernelemente sind in Abbildung 2 zusammengefasst.

Der Einstieg ins System erfolgt über eine benutzerspezifische Eingangsseite (Portal), auf der alle aktuellen Nachrichten, Termine und Dokumente auf einen Blick übersichtlich dargestellt sind. Die Ablage der Materialien wird über ein Dokumentenmanagement-System realisiert, das sich an den Erkenntnissen der CSCW-Forschung der letzten Jahrzehnte, aber vor allem an den schulspezifischen Bedürfnissen orientiert. Der Zugriff auf schulinterne Dokumente ist ebenso möglich wie das Herauf- und Herunterladen von Dokumenten, die zu Hause erstellt wurden. Dabei wurde eine Dateistruktur nach Klassen und Fächern vorgegeben, die sich allerdings jederzeit verändern lässt. Zum Austausch mit Gruppen oder Einzelpersonen werden spezielle Ordner zur Verfügung gestellt. Alle Dokumente lassen sich verschlagworten und mit Meta-Informationen nach den existierenden Standards versehen. Auf den Dokumenten lassen sich die gängigen Dateioperationen (Lesen, Schreiben, Löschen) je nach Rechten anwenden. 


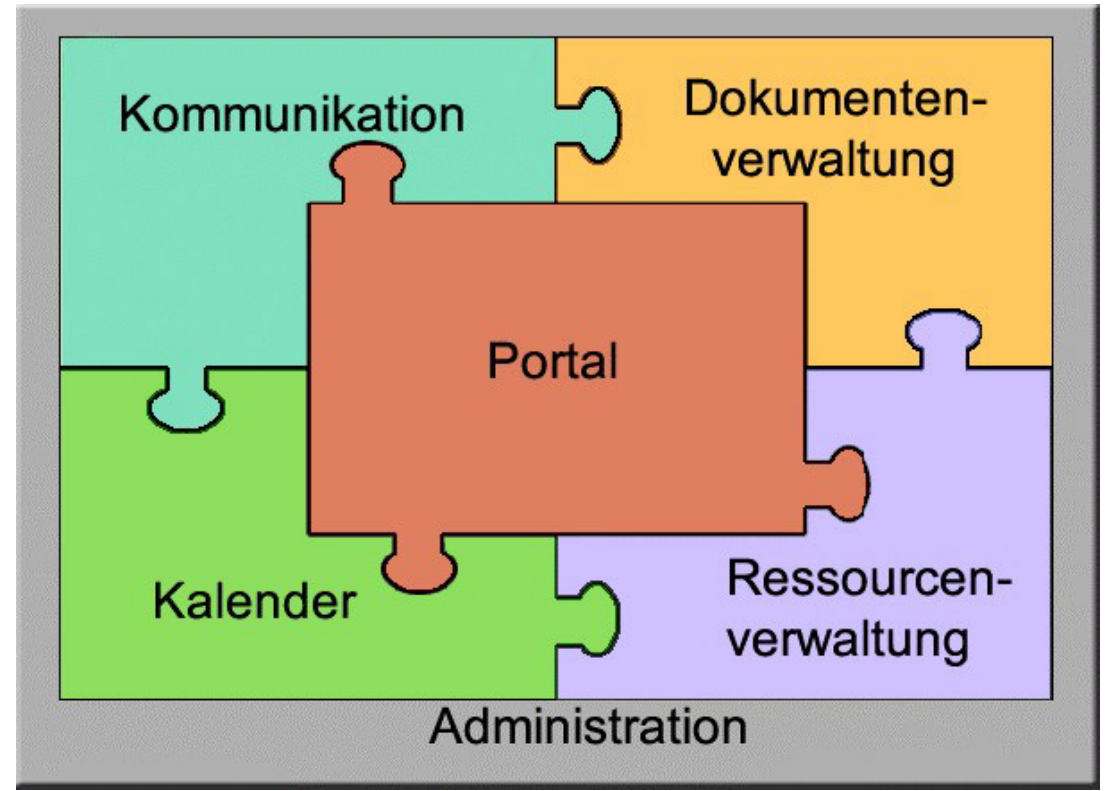

Abbildung 2: Komponenten des LearnWeb-Systems

Jedes verteilte System zur Unterstützung von Gruppenarbeit beinhaltet eine Palette der kommunikationsunterstützenden Werkzeuge wie E-Mail, Foren und Chats. Der Zugriff auf alle Werkzeuge und die Dokumente erfolgt über einen individualisierten Zugang, wie er mittlerweile bei den meisten «Community Plattformen» im Internet existiert (siehe MyYahoo, Web.de usw.). Dazu gehört auch ein Kalender für die eigenen Termine wie auch für schulbezogene Termine (Vertretungsplan, Projektwochen, Ausflüge) und Gruppentermine mit Benachrichtigungsfunktion (per Pop-up, SMS oder EMail).

Die Erfahrungen bei der Anforderungsanalyse aus den Schulen haben deutlich gemacht, dass erheblicher Bedarf an der technischen Unterstützung von planerischen Aufgaben besteht. Dazu gehören neben der Terminverwaltung (nicht die Stundenplanerstellung, dafür existieren eigene Programme, sondern für Terminveränderungen, Planung und Bekanntgabe von Projekttagen oder Sonderveranstaltungen) auch die Ressourcenverwaltung. In Schulen existiert erfahrungsgemäss nur eine eingeschränkte Zahl von mobilen Geräten (Beamer, Laptop, Rollwagen, Dia-/Film/Videoprojektor) und eine eingeschränkte Zahl von Zugangsorten für die
Arbeit mit Computer und dem Internet (Computerräume, Medienecken, Internet-Cafés). Noch sind nicht in allen Klassenräumen Anschlüsse und Geräte. Das System unterstützt daher auch die Planung der Nutzung von Räumen und Geräten.

Das Fundament des Systems bildet ein Administrationstool, das eine einfache dezentrale Nutzerverwaltung (Einrichten neuer und Löschen alter Nutzerinnen und Nutzer, jahrgangsweiser Wechsel der Klassen, Import von Daten aus dem Schulverwaltungssystem) ermöglicht und mit dem die Grundkonfiguration des Systems verändert werden kann. Auf die Realisierung des Autorentools wurde verzichtet, da eine intuitiv nutzbare Oberfläche für ungeübte Nutzerinnen und Nutzer im zeitlichen Rahmen und von der Komplexität nicht realisierbar erschien und für die geübten Lehrenden und Lernenden mächtige Standardwerkzeuge bereits zur Verfügung stehen.

\section{Erfahrungen in den Schulen}

Im Zuge mehrerer Iterationsschritte wurde zusammen mit Schülerinnen und Schülern sowie Lehrkräften der beteiligten Schulen Usability-Tests an verschiedenen Prototypen durchgeführt. Dabei kamen sowohl ExpertenReviews als auch nutzergestützte Tests entlang von komplexen Testaufgaben zum Einsatz. Die Ergebnisse dieser Tests wurden sowohl zur Verbesserung der Benutzungsschnittstelle als auch zur Fehlerbehebung bei den bestehenden Systemfunktionalitäten verwendet. Darüber hinaus wurden intensive Fortbildungsveranstaltungen in den Schulen angeboten und von den Studierenden durchgeführt, die auch für die Wartung und den Support zur Verfügung stehen.

Die Erfahrungen aus den Schulen mit der instrumentellen Nutzung des Systems wurden somit detailliert erhoben und werden im Zuge der Weiterentwicklung weiterhin berücksichtigt werden. Die Erfahrungen mit der organisatorischen Einbettung des Systems in die Strukturen und Abläufe der Schule sind bislang nur rudimentär erfasst worden. Hier besteht noch erheblicher Forschungsbedarf, um die Erkenntnisse, die bereits zur computerunterstützten Gruppenarbeit bzw. zur computervermittelten Kommunikation in anderen Bereichen (v. a. Unternehmen und Wissenschaft) existieren, auf Schulen und andere Bildungseinrichtungen zu übertragen. Aus den laufenden Erkenntnissen bei der Implementierung des Systems und den Ergebnissen aus vergleichbaren Projekten in anderen Schulen («School Wide Web» der Bertelsmann Stiftung in Paderborn, vom Gymnasium Ottobrunn und der Arbeitsgruppe «Intranet» des Netzwerks 
Medienschulen der Bertelsmann Stiftung) sowie in Verbindung mit den theoretischen Überlegungen zur Organisationsstruktur und der Ablauforganisation in Schulen lassen sich erste Hinweise auf Hindernisse bzw. Kriterien in drei Gruppen klassifizieren (vgl. Tabelle 2).

\begin{tabular}{|l|l|l|}
\hline $\begin{array}{l}\text { Technische } \\
\text { Kriterien }\end{array}$ & Organisatorische Kriterien & Personale Kriterien \\
\hline • stabile & $\bullet$ Einarbeitungszeit & $\bullet$ Medienkompetenz bei \\
technische & $\bullet$ Teamarbeit / Projektmanagement & Nutzerinnen und Nutzern \\
Infrastruktur & $\bullet$ Neue Arbeitsformen (kooperativ und & $\bullet$ Machtverlust \\
• einfach zu & virtuell) & (Einsichtnahme, \\
handhaben, & $\bullet$ Mehrfachverwendung (Verhinderung & Kontrolle) \\
• technische & von Abschreiben) & $\bullet$ Angst vor Blamage \\
Betreuung vor & $\bullet$ Wissensgewinnung & (Öffentlichkeit) \\
Ort & $\bullet$ Dokumentation (was ist sinnvoll zu & $\bullet$ Unsicherheit im Netz \\
$\bullet$ Plattformunab- & dokumentieren?, wer macht das? wie & (Datenschutz, \\
hängigkeit & geht das? wie aufwändig ist es?) & Datensicherheit) \\
- Barrierefreiheit & $\bullet$ Strukturierung der Inhalte & $\bullet$ Angst vor neuen \\
& $\bullet$ Anreizsystem & Arbeitsformen (Ende \\
& $\bullet$ Handlungsspielraum & eingeschliffener Routinen) \\
& $\bullet$ Restriktionen beim Zugriff / bei der & $\bullet$ Angst vor Verlust sozialer \\
& Veröffentlichung & Kontakte \\
\hline
\end{tabular}

Tabelle 2: Kriterien zur Bewertung des Einsatzes von Wissensmanagementsystemen

Betrachtet man die Modelle von Reinmann-Rothmeier bzw. von Probst und anderen (siehe Kapitel 2, Tabelle 1) so wird deutlich, dass in Schulen insbesondere Schwierigkeiten zwischen Wissenskommunikation und Wissensnutzung bzw. in den Phasen nach der Wissensentwicklung (hin zur Wissensverteilung, -nutzung, -bewahrung und -bewertung) bestehen. Die Wissensidentifikation und der Wissenserwerb bzw. Wissensgenerierung und -repräsentation stellen Lehrkräfte vor ähnliche Schwierigkeiten wie Unternehmen. Sehr viel komplexer wird die Frage nach der Wissensweitergabe und der Wissensnutzung. Die lose Kopplung der Organisationsmitglieder in der Schule in ihrer zellulären Struktur verhindern einen intensiveren Austausch, der zudem aufgrund der starken Fächerfokussierung und des geringen Anteils von projektbezogenem und fächerübergrei- fenden Unterricht basierend auf curricularen Bedingungen weiter erschwert wird.

Für die Wissensnutzung im Sinne eines produktiven Einsatzes organisationalen Wissens zum Nutzen der Organisation Schule im Alltag reicht die erfolgreiche Identifikation und (Ver-)Teilung zentraler Wissensbestandteile nicht aus. Für viele Menschen ist die Nutzung von Fähigkeiten oder Wissen fremder Wissensträger ein «widernatürlicher Akt» (Manago/Auriol 1996), den sie nach Möglichkeit vermeiden - das gilt insbesondere für Lehrkräfte in Schulen bei denen die Kooperations- und Kommunikationsbeziehungen noch nicht ausgeprägt sind. Gerade die Beibehaltung erprobter und gewohnter Routinen bildet einen Sicherheitsmechanismus, der den Einzelnen vor Informationsüberfluss schützt und seine Identität aufrechterhält. Organisationen müssen daher versuchen sicherzustellen, dass Wissen, das mit grossem Aufwand aufgebaut wird, auch alltagstauglich ist, und nicht das Beharrungsvermögen der Organisation und seiner Mitglieder überwiegt. Gerade die Qualität der eingestellten Daten und Informationen muss regelmässig überprüft werden, sonst landet man in der «Todesspirale der Daten» wie sie Manago und Auriol wie folgt beschreiben. «The data is not used because it is so difficult to assess, and no one invests in making it easy to retrieve because it is not used. The data is not trusted because many errors have been recorded, and no one cares to verify the accuracy of the data because it is not trusted» (Manago/Auriol 1996, S. 26).

Im Kern handelt es sich also beim Wissensmanagement um einen Prozess, der in die gesamte Organisationsentwicklung von Schulen eingebettet werden muss. Digitale Medien können hierfür einen Anstoss geben und die Organisationsmitglieder über die technische Faszination zum Mitwirken motivieren. Ohne organisatorische Absicherung und inhaltliche Ausgestaltung wird die Einführung eines solchen Systems wie auch in Unternehmen aber zum Scheitern verurteilt sein (vgl. Herrmann/Diefenbruch/Kienle 2002).

\section{Fazit}

Die Forschung zum Einsatz von technikunterstützten Wissensmanagementsystemen in Schulen stecken noch in den Kinderschuhen. Noch ist es zu früh, Ergebnisse aus den ersten Pilotschulen zu verallgemeinern. Die Zunahme an digitalem Unterrichtsmaterial und den Arbeitsergebnissen in digitaler Form von Schülerinnen und Schülern wird aber in vielen Schulen den Aufbau eines solchen Systems forcieren. Wegen der besonderen 
Organisationsstruktur von Schulen und des originären Organisationszweck von Lehren und Lernen lassen sich die Ergebnisse aus der arbeitswissenschaftlichen Forschung zu CSCW nicht übertragen.

Dennoch lohnt ein Blick in die Literatur, um spezifische Voraussetzung heraus zu destillieren. Zusammenfassend lassen sich zwei (vorläufige) Schlussfolgerungen ziehen:

(1) Wissensmanagement ist politisch und nur durch die Unterstützung der Schulleitung umsetzbar.

Das Wissensmanagement kann die Kompetenzen (Stärken und Schwächen) im Kollegium transparenter machen und möglicherweise auch verschieben. In diesem Prozess verlieren bisherige Fachexpertinnen und Fachexperten ihre besondere Position. Durch die Transparenz werden Informationsvorsprünge reduziert, die zuvor im Rahmen politischer Spiele durch die besser Informierten in der Schule genutzt werden. Konflikte haben eine negative Auswirkung auf die Nutzung kollaborationsunterstützender Technologien - in Schulen gibt es Gerangel um Ressourcen und traditionelle Streitigkeiten zwischen den Fachbereichen aufgrund der starken Fachspezialisierung bereits im Studium.

(2) Wissensmanagement muss in die Organisationsstruktur eingebette werden und die Schulkultur widerspiegeln.

Aufgrund der Arbeitsteilung im hierarchischen System der Schule und der auf der anderen Seite lose gekoppelten Einheiten von Klassenverbänden, Fachbereichen und Arbeitsgruppen lässt sich eine Querschnittsaufgabe wie das Wissensmanagement nur schwer etablieren. Um aber das vorhandene Wissen in der Organisation Schule besser zu nutzen, muss sich die Aufbau- und Ablauforganisation und die Schulkultur verändern. Wenn Anreize nur auf individueller Leistung basieren, ist die Wahrscheinlichkeit der Beteiligung an kollaborativen Aktivitäten niedriger - Lehrkräfte gelten vor allem in Deutschland als isolierte Einzelkämpferinnen und Einzelkämpfer. Das gesamte Kollegium muss bei der Bewältigung der «Informationsflut» unterstützt werden. Die Schule muss wegkommen vom gegenwärtig favorisierten, reproduktiven Lernen in traditionell strukturierten Klassenräumen und $\mathrm{zu}$ einer «Gemeinschaft mit Lernkultur»(Reinmann-Rothmeier/Mandl 1997, S. 56) verändert werden. Die Klasse als Lerngemeinschaft verstehen, die individuelle Kreativität, Eigeninitiative und Selbstverantwortung sowie Wissensaustausch, Zusammenarbeit und Gemeinsinn verstärkt.. Ein Form der strategischen Ausrichtung der Schule findet sich in einem Schulprogramm und dem dazugehörigen IT-Plan oder Medienkonzept (vgl. Breiter 2001).

\section{Literatur}

Argyris, C./Schön, D. A. (1978): Organizational Learning: a Theory of Action Perspective. Reading, MA.

Baitsch, C. (1993): Was bewegt Organisationen?: Selbstorganisation aus psychologischer Perspektive. Frankfurt/M.

Balzert, H. (1997): Lehrbuch der Software-Technik. Band 2. Heidelberg.

Beer, J. (2000): «Systemspezifikation.» In: Brössler, P./Siedersleben, J. (Hrsg.): Softwaretechnik. Praxiswissen für Software-Ingenieure. München, S. 21-49.

Bildungskommission-NRW (1995): Zukunft der Bildung - Schule der Zukunft: Denkschrift der Kommission «Zukunft der Bildung - Schule der Zukunft» beim Ministerpräsidenten des Landes Nordrhein-Westfalen. Neuwied.

Bødker, S./Grønbæk, K. (1991): «Design in Action: From Prototyping by Demonstration to Cooperative Prototyping.» In: Greenbaum, J./Kyng, M. (Hrsg.) (1991): Design at Work - Cooperative Design of Computer Systems. Hillsdale, NJ, S. 197-218.

Breiter, A. (2001): IT-Management in Schulen. Pädagogische Hintergründe, Planung, Finanzierung und Betreuung des Informationstechnikeinsatzes. Neuwied.

Bullinger, H.-J./Wörner, K./Prieto, J. (1998): «Wissensmanagement Modelle und Strategien für die Praxis.» In: Bürgel, H. D. (Hrsg.) (1998): Wissensmanagement: Schritte zum intelligenten Unternehmen. Berlin, S. 21-42.

Carroll, J. M. (Hrsg.) (1995): Scenario-Based Design: Envisioning Work and Technology in Systems Develpoment. New York.

Chott, P. O. (1998): Das Lehren des Lernens. In: PÄDForum, 26/11, 2, S. 174-180.

Cohen, M. D./March, J. G./Olsen, J. P. (1972): A Garbage Can Model of

Organizational Choice. In: Administrative Science Quarterly, 17, S. 1-25.

Crozier, M./Friedberg, E. (1979): Macht und Organisation: die Zwänge kollektiven Handelns. Königstein/Ts.

Davenport, T. H./Prusak, L. (1998): Wenn Ihr Unternehmen wüsste, was es alle weiss; das Praxisbuch, 2. Auflage. Landsberg/Lech. 
Dreyfus, H. L. (1992): What Computers still can't do. A critique of artifical reason. Cambridge, MA.

Geissler, H. (1994): Grundlagen des Organisationslernens. Weinheim.

Heinrich, L. J. (1999): Informationsmanagement - Planung, Überwachung und Steuerung der Informationsinfrastruktur, 6. Auflage. München/ Wien.

Herrmann, T./Diefenbruch, M./Kienle, A. (2002): Erfolgsfaktoren bei der Einführung von Wissensmanagementsystemen in die Praxis. In: Informatik Spektrum, 25, 3, S. 210-214.

Koschmann, T. D. (Hrsg.) (1996): CSCL: theory and practice of an emerging paradigm. Mahwah, NJ.

Krcmar, H. (1991): «Annäherungen an Informationsmanagement - Management und/oder Technologiedisziplin.» In: Staehle, W. H. / Sydow, J. (Hrsg.) (1991): Managementforschung. Berlin, S. 163-203.

Krcmar, H. (1996): Informationsmanagement. Heidelberg/New York.

Küpper, W./Ortmann, G. (Hrsg.) (1988): Mikropolitik. Rationalität, Macht und Spiele in Organisationen. Opladen.

Lortie, D. C. (1975): Schoolteacher: a sociological study. Chicago.

Manago, M./Auriol, E. (1996): Mining for Or. In: OR/MS Today, February, S. 28-32.

March, J. G./Olsen, J. P. (1986): «Garbage can models of decision making in organizations.» In: March, J.G./Weissinger-Baylon, R. (Hrsg.) (1986): Ambiguity and command. Marshfield, MA, S. 11-35.

Nonaka, I./Takeuchi, H. (1997): Die Organisation des Wissens: wie japanische Unternehmen eine brachliegende Ressource nutzbar machen. Frankfurt/Main.

Pedler, M./Boydell, T./Burgoyne, J. (1991): Auf dem Weg zum «Lernenden Unternehmen.»In: Sattelberger, T. (Hrsg.) (1991): Die lernende Organisation: Konzepte für eine neue Qualität der Unternehmensentwicklung. Wiesbaden.

Polanyi, M. (1966): The Tacit Dimension. London.

Probst, G./Raub, S./Romhardt, K. (1997): Wissen managen: wie Unternehmen ihre wertvollste Ressource optimal nutzen. Wiesbaden.

Pulic, A. (1996): Der Informationskoeffizient als Wertschöpfungsmass wissensintensiver Unternehmungen. In: Schneider, U. (Hrsg.) (1996): Wissensmanagement: die Aktivierung des intellektuellen Kapitals. Frankfurt/M., S. 147-180.

Reinmann-Rothmeier, G./Mandl, H. (1997): «Wissensmanagement in der
Bildung.» In: Höfling, S./Mandl, H. (Hrsg.) (1997): Lernen für die Zukunft - Lernen in der Zukunft. Wissensmanagement in der Bildung. München, S. 56-66.

Reinmann-Rothmeier, G./Mandl, H. (1999): Wissensmanagement: Modewort oder Element der lernenden Organisation? In: Personalführung, 22, 12, S. 18-23.

Rolff, H.-G. (1993): Wandel durch Selbstorganisation. Theoretische Grundlagen und praktische Hinweise für eine bessere Schule. München.

Rolff, H.-G. u.a. (1998): Manual Schulentwicklung. Handlungskonzepte zur pädagogischen Schulentwicklungsberatung (SchuB). Weinheim/Basel.

Schulmeister, R. (2000): Selektions- und Entscheidungskriterien für die Auswahl von Lernplattformen und Autorenwerkzeugen. GUTACHTEN für das BM:BWK. Universität Hamburg. www.izhd.uni-hamburg.de/ pdfs/Plattformen.pdf.

Schüppel, J. (1996): Wissensmanagement: organisatorisches Lernen im Spannungsfeld zwischen Wissens- und Lernbarrieren. Wiesbaden.

Senge, P. M. (1996): Die fünfte Disziplin: Kunst und Praxis der lernenden Organisation. Stuttgart.

Suchman, L. A. (1987): Plans and Situated Actions. Cambridge, MA.

Synnott, W. R./Gruber, W. H. (1981): Information resource management: opportunities and strategies for the 1980s. New York.

Warnken, G. (1997): Das Schulprogramm. In: schul-management, 28, 5, S. $18-28$.

Weick, K. E. (1976): Educational Organizations as Loosely Coupled Systems. In: Administrative Science Quarterly, 21, S. 1-19.

Wollnik, M. (1989): «'Informationsmanagement' - Szenenwechsel in der computergestützten Informationsverarbeitung und die Zukunft der Endbenutzer.» In: Ortmann, G./Windeler, A. (Hrsg.) (1989): Umkämpftes Terrain. Managementperspektiven und Betriebsratspolitik bei der Einführung von Computer-Systemen. Opladen, S. 51-99. 